\section{Andes \\ EDIATRICA \\ Revista Chilena de Pediatría}

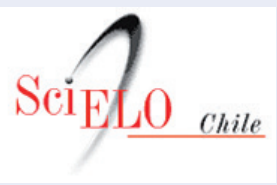

www.scielo.cl

\title{
Apendicitis aguda complicada en pacientes pediátricos con antecedentes de "empacho" y manipulación abdominal: serie de casos
}

\section{Complicated acute appendicitis in pediatric patients with "empacho" and abdominal manipulation history: case series}

\author{
David Aguilar-Andino, ${ }^{\mathrm{a}, \mathrm{f}}$, Tania Soledad Licona Rivera ${ }^{\mathrm{b}, \mathrm{g}}$, Jorge Alberto Osejo Quan ${ }^{\mathrm{a}}$, \\ Ricardo Jafet Carranza Linares ${ }^{\mathrm{a}, \mathrm{h}}$, Marco Antonio Molina Soto ${ }^{\mathrm{c}, \mathrm{d}}$, César Alas-Pineda $^{\mathrm{d}, \mathrm{e}, \mathrm{i}}$
}

\author{
aDepartamento de Medicina, Universidad Nacional Autónoma de Honduras. Valle de Sula, Cortés, Honduras. \\ bDepartamento de Pediatría, Universidad Nacional Autónoma de Honduras. San Pedro Sula, Honduras. \\ 'Departamento de Postgrado de Medicina Interna, Universidad Nacional Autónoma de Honduras. San Pedro Sula, Honduras. \\ dHospital Dr. Mario Catarino Rivas. San Pedro Sula, Honduras \\ eUniversidad Católica de Honduras. San Pedro Sula, Honduras \\ fhttps://orcid.org/0000-0001-6750-4092 \\ ghttps://orcid.org/0000-0003-4348-7181 \\ hhttps://orcid.org/0000-0003-0813-2289 \\ ihttps://orcid.org/0000-0003-4183-9104
}

Recibido: 1 de octubre de 2020; Aceptado: 9 de diciembre de 2020

¿Qué se sabe del tema que trata este estudio?

La apendicitis aguda es una emergencia quirúrgica que amerita ser tratada con prontitud. La demora del diagnóstico y tratamiento conlleva a sus diversas complicaciones. El empacho se conoce como un síndrome folclórico con un cuadro clínico descrito muy variable.

\section{¿Qué aporta este estudio a lo ya conocido?}

La creencia del empacho tiene una influencia indirecta en el deterioro clínico de la apendicitis aguda mediante el retraso diagnóstico y terapéutico de la misma, lo cual contribuye a la generación de diversas complicaciones ya conocidas.

\section{Resumen}

Según la cultura popular latinoamericana, el empacho es una enfermedad intestinal causada por ingesta excesiva de alimentos, fríos o mal cocidos. La manipulación abdominal o "sobada" es uno de los tratamientos populares utilizados para el empacho y consiste en un masaje abdominal, donde se frota, se estira la piel y se aplica presión sobre el abdomen. Objetivos: Caracterizar la evolución clínica y las complicaciones secundarias al retraso diagnóstico, en pacientes pediátricos con apendicitis
Palabras clave: Apendicitis; Apendicitis Perforada; Etnografía; Medicina Popular; Medicina de Emergencia; Pediatría 
aguda, con antecedente de empacho y manipulación abdominal. Pacientes y Método: Estudio observacional descriptivo, prospectivo de pacientes pediátricos con apendicitis aguda complicada con antecedente de manipulación abdominal, captados en la sala de cirugía pediátrica entre noviembre del 2019 y junio del 2020 en el Hospital Mario Catarino Rivas. Se empleó estadística descriptiva para el análisis. Resultados: Se estudiaron 10 pacientes, rango de edad 6 a 16 años (mediana 14 años), 50\% procedente de zonas rurales. Los principales síntomas que se presentaron previo a la manipulación abdominal fueron: vómitos, náuseas y anorexia, posterior a lo cual se agregó diarrea espontanea o estreñimiento y aumento de la intensidad del dolor. Las complicaciones fueron perforación intestinal $(5 / 10)$, plastrón apendicular (3/10), absceso intraabdominal $(3 / 10)$, y apéndice en fase gangrenosa (1/10). La mediana de tiempo entre síntoma inicial y hospitalización fue 60 horas [IQR, 33,0-72,0]. Conclusión Se describe la influencia del empacho y su respectivo tratamiento en el deterioro clínico debido al retraso diagnóstico y del tratamiento de la apendicitis aguda, dando como resultado distintas complicaciones.
Keywords: Appendicitis; Perforated Appendicitis; Ethnography; Traditional Medicine; Emergency Medicine; Pediatrics

\section{Introducción}

La apendicitis se define como la inflamación del apéndice vermiforme (en forma de gusano), una extensión estrecha en forma de bolsa, de terminación ciega, aproximadamente 5 a $9 \mathrm{~cm}$ de largo de la apertura del ciego ${ }^{1,2}$. En el primer año de vida, el apéndice tiene una morfología en embudo, con baja predisposición a la obstrucción, debido a que los folículos linfoides que pueden obstruir el apéndice alcanzan un tamaño máximo durante la adolescencia, no obstante, la apendicitis infantil es la principal etiología de abdomen agudo en niños en edad preescolar y escolar a nivel mundial ${ }^{3}$.

La patogénesis exacta de la apendicitis aguda es multifactorial, siendo la obstrucción de la luz proximal la principal causa, generada por inflamación de la mucosa, hiperplasia linfoide o un fecalito, desencadenando una acumulación de las secreciones mucosas del tramo distal, con un aumento de la presión intraluminal, produciendo un colapso del sistema venoso y su trombosis, con inflamación transmural supurativa. Si la obstrucción persiste, la congestión condiciona un estado isquémico con gangrena y finalmente perforación del apéndice ${ }^{4-6}$.

Clásicamente, el cuadro clínico de apendicitis se ha descrito como la aparición gradual de dolor periumbilical sordo que migra al cuadrante inferior derecho. Este cuadro normalmente se acompaña de síntomas adicionales como náuseas, vómitos, anorexia, fiebre y con menor frecuencia diarrea ${ }^{7}$. La presentación clínica no específica en niños menores de 5 años, así como dificultades para comunicarse con ellos, examen físico inadecuado, irritabilidad y superposición de los síntomas con otras enfermedades infantiles comunes se atribuyen al diagnóstico tardío de apendicitis aguda y alta tasa de diagnóstico erróneo ${ }^{8}$.

La clínica insidiosa de la apendicitis aguda en pa- 
cientes pediátricos, el desconocimiento de la patología y las creencias socioculturales son todos factores que pueden influenciar a los padres y/o responsables a considerar el empacho como diagnóstico en niños con un cuadro de apendicitis aguda. En la historia médica latinoamericana existen reportes sobre el empacho desde el siglo XVI, convirtiéndolo en una enfermedad reconocida dentro de las creencias populares ${ }^{9}$. Dicha enfermedad ha demostrado una continuidad histórica y un patrón de transmisión cultural generacional en toda Latinoamérica. Estos conocimientos populares tienen orígenes indígenas americanos y europeos mediterráneos, indisolublemente fusionados ${ }^{10,11}$.

En un estudio de revisión realizado por CamposNavarro R. (2016), se define el empacho como una indigestión intestinal, generalmente con diarrea, que afecta sobre todo a lactantes y a niños de corta edad, presentándose principalmente en los niños tras comer alimentos mal cocidos o fríos ${ }^{11}$. El cuadro clínico del empacho se asemeja a un cuadro clásico de apendicitis aguda, debido a esto muchos padres acuden a sobadores o familiares para dar tratamiento al cuadro clínico del empacho, que consiste en remedios naturales herbales y manipulación abdominal ${ }^{12}$.

La manipulación abdominal, también conocida como "sobada", consiste en una especie de masaje abdominal, en el cual se aplica frotación, estiramiento de la piel y presión sobre el abdomen del paciente; para tratar de curar el síndrome de empacho ${ }^{13}$. Los terapeutas, frecuentemente utilizan aceites o manteca para facilitar las maniobras ${ }^{14}$, y después de dicho procedimiento; se administra una cucharada de aceite de oliva, purgantes y/o la toma de un té, donde se utiliza una variedad entre 17 y 39 especies de hierbas para su elaboración ${ }^{15,16}$.

Sin embargo, cuando se trata de un cuadro de apendicitis aguda, la manipulación abdominal contribuye al retraso diagnóstico, modificando el cuadro clínico y dificultando al cirujano su identificación, dando como resultado las posibles complicaciones de la apendicitis $\operatorname{aguda}^{17}$.

El objetivo de este estudio es caracterizar la evolución clínica y las complicaciones en una serie de pacientes pediátricos diagnosticados con apendicitis aguda complicada, y con antecedentes de manipulación abdominal por sospecha de empacho.

\section{Pacientes y Método}

Estudio observacional, descriptivo, prospectivo, tipo serie de casos. Se analiza una serie de 10 pacientes pediátricos post operados por apendicitis aguda complicada, los cuales tenían el antecedente de manipulación abdominal, captados en la sala de cirugía pediátrica entre noviembre del 2019 y junio del 2020, en el Hospital Nacional Dr. Mario Catarino Rivas, un hospital de segundo nivel de atención que sirve como centro de mayor referencia para toda la zona noroccidental de Honduras.

Se incluyeron pacientes pediátricos con diagnóstico de apendicitis aguda sometidos a manipulación abdominal por personal no médico, debido a sospecha de empacho. Se excluyeron cuadros de abdomen agudo de etiología diferente a apendicitis aguda y apendicitis simple como en el caso de 3 pacientes con el antecedente de manipulación abdominal y diagnóstico postoperatorio de apendicitis simple. Se enroló al total de pacientes pediátricos que cumplía con criterios de inclusión cuya historia clínica confirmaba la manipulación abdominal.

Se elaboró un formulario de recolección de las variables con validez de contenido por expertos. Se tomó en estudio el cuadro clínico consignado en el expediente al ingreso (sintomatología, tiempo de evolución e intensidad del dolor y estancia hospitalaria), variables sociodemográficas: edad, sexo, zonificación (rural y urbana), escolaridad paterna ( $\sin$ escolaridad, primaria, secundaria y educación superior), exámenes de laboratorio (hemograma, PCR), y el cuadro clínico previo y posterior a la manipulación abdominal mediante una entrevista directa al paciente. Se aplicaron escalas acordes a la edad del paciente para cuantificar la variable de intensidad del dolor abdominal, aplicando la escala visual para el dolor de Wong-Baker en pacientes con edades entre 3 a 10 años ${ }^{18}$. En pacientes con edades de 10 a 18 años se utilizó una escala numérica análoga del dolor para cuantificar dicha intensidad.

La data fue ingresada, en el Programa Estadístico IBM SPSS versión 25.0 (licencia de uso en vigencia), en donde se realizó el análisis estadístico. Se empleó estadística descriptiva, se obtuvo las frecuencias y porcentajes de las variables categóricas, y se trabajó con la mediana y rangos intercuartílicos de las variables cuantitativas.

El proyecto fue aprobado por el comité de ética institucional de la Universidad Nacional Autónoma de Honduras con numero \#003-04. Tras la aprobación del protocolo del estudio, se procedió a la captación activa de pacientes en sala de cirugía pediátrica, se solicitó consentimiento informado firmado por los padres o tutores y asentimiento del paciente pediátrico previo al enrolamiento.

\section{Resultados}

Se analizó una muestra de 10 pacientes pediátricos, con apendicitis aguda complicada que fueron sometidos a manipulación abdominal. La mediana de edad 
fue de 14 años [IQR,11,8-15,0 años] con un rango que oscila entre 6 y 16 años. El grupo etario de 13-14 años presentaba la mayoría de casos (4/10), seguido del grupo de 15-16 años (3/10). Seis de los pacientes incluidos en estudio pertenecen al sexo masculino. La mitad de los pacientes procedían de zonas rurales. Respecto al grado académico del padre o tutor responsable del paciente, seis de ellos habían culminado la primaria completa, uno sin escolaridad, y tres padres con educación superior completa (tabla 1 ).

El dolor abdominal fue el síntoma inicial en 9 de los pacientes incluidos en la serie, solo un paciente presentó fiebre como sintomatología inicial. La mediana de la intensidad dolor fue de 5 puntos [IQR, 5,0-6,5 puntos] en la escala análoga del dolor. Se reportaron dos pacientes con una intensidad de 8/10 en dicha escala. Todos los pacientes cursaron con dolor abdominal en su cuadro clínico, 9 de ellos localizados en el cuadrante inferior derecho, 9 pacientes refirieron haber tenido fiebre subjetivamente alta en las primeras horas del cuadro de dolor (tabla 2). Los principales síntomas que se presentaron previo a la manipulación abdominal fueron vómitos $(10 / 10)$, náuseas $(9 / 10)$, anorexia (8/10), signo de Dunphy también conocido como el dolor abdominal al toser (8/10) e hipersensibilidad abdominal (7/10). La mediana de tiempo entre la aparición del síntoma inicial y la manipulación abdominal, fue de $24 \mathrm{~h}$ [IQR 19-30 h].

Posterior a la manipulación abdominal, los pacientes refirieron un deterioro clínico gradual, y aumento de la intensidad del dolor (7/10), presentándose generalización del dolor abdominal en los cuatro cuadrantes (6/10). Aumento de la intensidad del dolor en la escala análoga, con una mediana de 8 puntos [IQR, 6,5-10]. Se agregó diarrea (4/10) y es- treñimiento (4/10), manteniéndose constantes la presencia de náuseas $(9 / 10)$, vómitos $(10 / 10)$ y anorexia (8/10) (tabla 3).

Al momento de la hospitalización, 9 pacientes presentaron signo de McBurney (dolor a la palpación abdominal causado por la aplicación de presión entre $1 / 3$ externo con los $2 / 3$ internos de una línea imaginaria trazada entre la espina iliaca antero-superior derecha hasta el ombligo) y signo de rebote positivos (dolor a la descomprensión abdominal).

En el hemograma destacó leucocitosis de 10.000$15.000 / \mathrm{mm}^{3}(3 / 10), 15.000-20.000 / \mathrm{mm}^{3}(2 / 10)$ y superior a $20.000 / \mathrm{mm}^{3}(3 / 10), 2$ casos presentaron valores de leucocitos inferiores a $10.000 / \mathrm{mm}^{3}$. Entre los exámenes complementarios destaca Proteína C Reactiva en rango de normalidad en 9 pacientes y examen general de orina sin alteraciones. No se realizaron estudios de imagen en los pacientes analizados en este estudio, debido a la emergencia quirúrgica que estos presentaban.

Todos los pacientes fueron intervenidos quirúrgicamente en las primeras horas del diagnóstico. Las complicaciones más frecuentes encontradas fueron: perforación intestinal $(5 / 10)$, plastrón apendicular (3/10), absceso intraabdominal (3/10), y otras complicaciones como apéndice en fase gangrenosa (1/10) (tabla 2). El caso $n^{\circ} 7$ presentó tres complicaciones consecutivas: perforación intestinal, plastrón apendicular y el desarrollo de un absceso intraabdominal.

La estancia hospitalaria en todos los pacientes tuvo un promedio de 7-14 días, debido a las diferentes complicaciones encontradas. La mediana de tiempo entre el síntoma inicial y la hospitalización fue $60 \mathrm{~h}$ [IQR, $33,0-72,0]$, lo que equivale a más de dos días y medio para acudir a un centro de atención hospitalaria.

Tabla 1. Características sociodemográficas de 10 pacientes con apendicitis aguda complicada

\begin{tabular}{ccccc}
\hline$N^{\circ}$ paciente & Sexo & Edad & Procedencia & Escolaridad de responsable \\
\hline 1 & Mujer & 16 años & Rural & Primaria \\
2 & Hombre & 14 años & Urbana & Analfabeta \\
3 & Mujer & 6 años & Urbana & Primaria \\
4 & Hombre & 11 años & Rural & Primaria \\
5 & Hombre & 14 años & Urbana & Primaria \\
6 & Hombre & 14 años & Urbana & Unimaria \\
7 & Mujer & 14 años & Urbana & Primaria \\
8 & Hombre & 15 años & Rural & Universitaria \\
\hline
\end{tabular}


Tabla 2. Manifestaciones clínicas de 10 pacientes con apendicitis aguda complicada

\begin{tabular}{|c|c|c|c|c|c|c|c|c|}
\hline $\begin{array}{c}\mathrm{N}^{\circ} \\
\text { paciente }\end{array}$ & $\begin{array}{l}\text { Síntoma } \\
\text { inicial }\end{array}$ & $\begin{array}{l}\text { Tiempo de } \\
\text { evolución } \\
\text { hasta la } \\
\text { hospitalización }\end{array}$ & $\begin{array}{l}\text { Intensidad } \\
\text { del dolor } \\
\text { previa } \\
\text { manipulación }\end{array}$ & $\begin{array}{l}\text { Intensidad } \\
\text { de dolor } \\
\text { posterior a } \\
\text { manipulación }\end{array}$ & $\begin{array}{c}\text { Generalización } \\
\text { del dolor } \\
\text { abdominal }\end{array}$ & $\begin{array}{l}\text { Leucocitos } \\
\text { al ingreso } \\
\text { cel } / \mathrm{mm}^{3}\end{array}$ & $\begin{array}{l}\text { Complicaciones } \\
\text { encontradas }\end{array}$ & $\begin{array}{l}\text { Estancia } \\
\text { hospitalaria } \\
\text { (días) }\end{array}$ \\
\hline 1 & Dolor & $96 \mathrm{~h}$ & 5 & 10 & + & $10-15,000$ & Perforación intestinal & 7-14 días \\
\hline 2 & Dolor & $36 \mathrm{~h}$ & 5 & 8 & + & $<10,000$ & Perforación intestinal & 3-7 días \\
\hline 3 & Dolor & $72 \mathrm{~h}$ & 5 & 10 & - & $10-15,000$ & Absceso intrabdominal & 3-7 días \\
\hline 4 & Dolor & $24 \mathrm{~h}$ & 8 & 8 & - & $15-20,000$ & $\begin{array}{c}\text { Apendicitis en fase } \\
\text { gangrenosa }\end{array}$ & 3-7 días \\
\hline 5 & Dolor & $48 \mathrm{~h}$ & 5 & 5 & + & $10-15,000$ & Perforación intestinal & 3-7 días \\
\hline 6 & Dolor & $72 \mathrm{~h}$ & 5 & 10 & + & $>20,000$ & Plastrón apendicular & 3-7 días \\
\hline 7 & Dolor & $72 \mathrm{~h}$ & 5 & 7 & + & $>20,000$ & $\begin{array}{l}\text { Perforación intestinal, } \\
\text { plastrón apendicular, } \\
\text { absceso intraabdominal }\end{array}$ & $>14$ días \\
\hline 8 & Fiebre & $24 \mathrm{~h}$ & 8 & 10 & + & $15-20,000$ & Plastrón apendicular & 3-7 días \\
\hline 9 & Dolor & $48 \mathrm{~h}$ & 5 & 5 & - & $<10,000$ & Absceso intraabdominal & 7-14 días \\
\hline 10 & Dolor & $72 \mathrm{~h}$ & 6 & 8 & - & $>20,000$ & Perforación intestinal & 7-14 días \\
\hline
\end{tabular}

aEscala análoga del dolor (1-10 puntos).

Tabla 3. Manifestaciones clínicas previa y posterior manipulación abdominal de los 10 pacientes con apendicitis aguda complicada

\begin{tabular}{lcc}
\hline Manifestaciones clínicas & $\begin{array}{c}\text { Previa } \\
\text { manipulación } \\
\text { abdominal }^{\mathrm{a}}\end{array}$ & $\begin{array}{c}\text { Posterior a } \\
\text { manipulación } \\
\text { abdominal }^{\mathrm{a}}\end{array}$ \\
\hline $\begin{array}{l}\text { Intensidad del dolor, mediana } \\
\text { Leve }\end{array}$ & 0 & $\begin{array}{c}\text { [IQR, 5,0-6,5] } \\
\text { [IQR, 6,5-10] }\end{array}$ \\
Moderado & 8 & 2 \\
Severo & 2 & 7 \\
Dolor generalizado & 1 & 6 \\
Si & 9 & 4 \\
No & & \\
Sintomatología & 9 & 9 \\
Náuseas & 10 & 10 \\
Vómitos & 8 & 8 \\
Anorexia & 0 & 4 \\
Diarrea & 0 & 4 \\
Estreñimiento & 9 & 9 \\
Fiebre & 8 & 9 \\
Signo de Dunphy & 7 & \\
Sensibilidad a la palpación & & \\
\hline
\end{tabular}

a La información se obtuvo de la totalidad de los pacientes $(n=10)$.

\section{Discusión}

El dolor abdominal es uno de los principales síntomas de los pacientes pediátricos atendidos en las emergencias, y la apendicitis representa la emergencia quirúrgica abdominal más común en esta población ${ }^{19}$, más aun cuando el paciente se ve expuesto a factores externos de manipulación abdominal que pudiesen retrasar el diagnóstico e influir en el deterioro clínico.

Podany AB. et al (2017) reporta que la tasa de perforación en la población pediátrica disminuye a medida aumenta la edad, estableciendo tasas de casi el 100\% en paciente de un año de vida; 50-69\% a la edad de 5 años y en los adolescentes del $10-20 \%{ }^{20}$. En este estudio, el grupo etario más afectado fueron los adolescentes mayores de 14 años, esto contrasta con lo reportado en la literatura.

Unos de los factores posiblemente implicados en el aumento de la incidencia de apendicitis complicada, pocas veces tomado en cuenta, son las creencias socioculturales. El empacho es una enfermedad culturalmente popular que no es reconocida por el gremio médico, pero sí por todo el argot popular en Latinoamérica y por los grupos hispanos de los Estados Unidos de Norteamérica. Los síntomas descritos con frecuencia son gastrointestinales como: distensión abdominal, indigestión, diarrea, vómitos, letargo, repugnancia a los alimentos, y en ocasiones estreñimiento ${ }^{21}$. Otros síntomas no gastrointestinales descritos son: malestar general, postración, fiebre, sudoración, lengua seca, y blanquecina o con mucosidades amarillentas ${ }^{10}$. En esta serie de casos, la mayoría de los pacientes presentaron un cuadro clínico similar con: dolor abdominal (100\%), vómitos (100\%), náuseas (90\%), fiebre (90\%), y anorexia $(80 \%)$. 
El cuadro de apendicitis aguda suele ser confundido, generalmente por los padres y/o responsables, con un episodio de empacho, debido a la similitud de las manifestaciones clínicas popularmente descritas. Es por ello que muchos padres y/o responsables de los pacientes deciden acudir a un familiar o terapeuta comúnmente conocido como "sobador", quien realiza tratamientos caseros naturales y procedimientos manuales. Dentro de los procedimientos manuales, se encuentra la manipulación abdominal.

En la serie de casos presentada, todos los padres y/o responsables acudieron a un terapeuta o "sobador", siendo este un familiar o un particular, por la sospecha de un cuadro de empacho. En un estudio cualitativo realizado por Sandberg JC, et al. (2018), se entrevistó a 24 inmigrantes mexicanos, y se estableció que los participantes percibían que los médicos son profesionales que se dedican a las enfermedades biomédicas crónicas y agudas, preferidos para las heridas y muchas patologías internas, también refieren que no perciben al personal médico suficientemente eficaces para tratar enfermedades de base cultural arraigadas, como el empacho $0^{22}$.

En este estudio se demuestra el deterioro clínico de los pacientes que evolucionaron con generalización y aumento de la intensidad del dolor abdominal. La evolución con diarrea posterior a la "soba" hizo sospechar en algunos casos de una gastroenteritis, diagnóstico erróneo más común de los pacientes con apendicitis, de hecho, la diarrea puede estar presente en $33-41 \%{ }^{23}$.

El modelo fisiopatológico convencional de la apendicitis aguda se basa en una relación entre el tiempo y la progresión de la enfermedad. Los retrasos pueden ocurrir en cualquier lugar a lo largo del camino desde el inicio de los síntomas hasta la presentación, evaluación y tratamiento ${ }^{24}$.

En estudios realizados por Marmo AS et al. (2014) y por Amin P et al. (2012), mencionan que la prevalencia de perforación es de un 7\% cuando los síntomas están presentes en menos de $24 \mathrm{~h}$, y aumenta exponencialmente a $98 \%$, cuando los síntomas están presentes más de $48 \mathrm{~h}^{25,26}$. Los hallazgos en este estudio, respecto al tiempo de evolución del inicio de los síntomas, y la hospitalización fueron superiores a 48 h en 8 de los casos, concordando con lo mencionado. Dos de los casos tuvieron un tiempo de evolución más corto, por lo cual se podrían considerar factores externos, que pudiesen modificar drásticamente el curso de la enfermedad.

En este estudio, el promedio de estancia hospitalaria en los diez pacientes fue de 7-14 días, concordando con Chen CL. et al., (2017) realizado para identificar factores de riesgo de hospitalización prolongada en pacientes pediátricos con apendicitis que no se sometieron inicialmente a tratamiento quirúrgico, en el cual la mediana de estancia hospitalaria fue de 14 días $^{27}$.
El retraso en el diagnóstico sigue siendo uno de los principales factores de riesgo para la complicación de la apendicitis aguda. En un estudio multicéntrico Pedziwiatr M. et al., (2019) demostraron que una prolongación entre el inicio de los síntomas y la atención hospitalaria es un factor de riesgo para apendicitis aguda complicada. Así mismo, en un estudio de cohortes de 115 pacientes realizado por Khan J. et al. (2018), se identificaron las distintas causas del retraso en el diagnóstico de apendicitis agudas, encontrando que el mayor factor contribuyente fue el mal manejo y diagnóstico por curanderos en un $32,17 \%$ y por doctores en un $26 \%{ }^{28}$.

De igual forma, se debe tomar en consideración, que las diferentes maniobras implementadas por el terapeuta pudiesen ser uno de los factores que contribuyen a la perforación del apéndice, debido al probable aumento de la presión intraabdominal. Similar a lo propuesto en el estudio de Zvizdic Z. et al. (2020), en un caso de apendicitis perforada secundario a trauma abdominal cerrado, establece que el aumento súbito de la presión intraabdominal puede provocar un cuadro de apendicitis aguda ${ }^{29}$. En esta serie de casos, en que existe un cuadro de apendicitis aguda de tipo obstructiva ya instaurada, el aumento de la presión intraabdominal, podría influir en el colapso vascular y en el aumento de la presión intracecal, dando como resultado a la perforación del apéndice.

La alta incidencia de apendicitis complicada en presencia del antecedente de manipulación abdominal, popularmente realizada para aliviar y/o curar el empacho, deben ser estudiadas a mayor profundidad y rigurosidad, con una mayor población de pacientes, para establecer la posible relación causal entre estas dos variables.

\section{Conclusión}

Las creencias socioculturales, como el empacho, juegan un papel importante en la evolución y pronóstico del paciente con apendicitis aguda en Latinoamérica. El empacho, y su respectiva manipulación abdominal como tratamiento empírico, influyen en el deterioro clínico del paciente, aumentando la intensidad y la generalización del dolor abdominal, incurriendo en el retraso del diagnóstico y del manejo adecuado de la apendicitis. Lo anterior daría como resultado las distintas complicaciones.

Se recomienda al personal médico comunitario realizar concientización en la población general, sobre el abdomen agudo y de esta manera combatir la creencia popular del empacho, evitar la práctica de su respectivo tratamiento y favorecer la consulta oportuna. 


\section{Responsabilidades Éticas}

Protección de personas y animales: Los autores declaran que los procedimientos seguidos se conformaron a las normas éticas del comité de experimentación humana responsable y de acuerdo con la Asociación Médica Mundial y la Declaración de Helsinki.

Confidencialidad de los datos: Los autores declaran que han seguido los protocolos de su centro de trabajo sobre la publicación de datos de pacientes.

Derecho a la privacidad y consentimiento informado: Los autores han obtenido el consentimiento in- formado de los pacientes y/o sujetos referidos en el artículo. Este documento obra en poder del autor de correspondencia.

\section{Conflicto de intereses}

Los autores declaran no tener conflicto de intereses.

\section{Agradecimientos}

Los autores agradecen a la Dra. Alessandra Pazzetti por la ayuda en la revisión y recolección de información de los expedientes clínicos de los pacientes.

\section{Referencias}

1. Sellars H, Boorman P. Acute appendicitis. Surgery (Oxford) 2017;35(8):432-8.

2. Gadiparthi R, Waseem M. Pediatric Appendicitis. [Actualizado el 5 de julio de 2020]. In: StatPearls [Internet]. Treasure Island (FL): StatPearls Publishing; 2020 Jun. Disponible en: https://www.ncbi.nlm. nih.gov/books/NBK441864/

3. Sakellaris G, Partalis N, Dimopoulou D. Apendicitis en niños de edad preescolar. Salud(i) Ciencia 2015;(21):284-93.

4. Baird DLH, Simillis C, Kontovounisios C, Rasheed S, Tekkis PP. Acute appendicitis. BMJ 2017;357:j1703.

5. Stringer MD. Acute appendicitis. J Paediatr Child Health. 2017;53(11):10716.

6. Souza-Gallardo LM, Martínez-Ordaz JL. Apendicitis aguda. Manejo quirúrgico y no quirúrgico. Rev Med Inst Mex Seguro Soc. 2017;55(1):76-81.

7. Rentea RM, St Peter SD. Pediatric Appendicitis. Surg Clin North Am. 2017;97(1):93-112.

8. Almaramhy HH. Acute appendicitis in young children less than 5 years: review article. Ital J Pediatr. 2017;43(1):15.

9. El empacho: una enfermedad popular latinoamericana. Cuad Hist Salud Pública [Internet] 2007 [citado 2020 Nov 15];102. Disponible en: http://scielo.sld.cu/scielo. php?script=sci_arttext\&pid=S004591782007000200004\&lng=es.

10. Campos R. El empacho: una enfermedad popular en infantes del Uruguay. Rev. urug. antropología etnografía. 2017;2(2):45-60

11. Campos Navarro R. El empacho: revisión de una enfermedad popular infantil chilena (1674-2014). Rev Chil Pediatr. 2016;87(1):63-8.

12. Campos-Navarro R, Scarpa GF. The cultural-bound disease "empacho" in
Argentina. A comprehensive botanicohistorical and ethnopharmacological review. J Ethnopharmacol. 2013;148(2):349-60.

13. Graham A, Sandberg JC, Quandt SA, Mora DC, Arcury TA. Manual Therapy Practices of Sobadores in North Carolina. J Altern Complement Med. 2016;22(10):841-6.

14. Espinosa-Cortés LM, HernándezMartínez BA. Empacho y prácticas terapéuticas en la costa chica de Guerrero y Oaxaca. Rev Invest Clin. 2012;64(6 Pt 1):576-85.

15. Urióstegui-Flores A. Síndromes de filiación cultural atendidos por médicos tradicionales. Rev Salud Pública (Bogota) 2015;17(2):277-88.

16. Pérez-Ochoa M, Chávez-Servia J, Vera-Guzmán A, Aquino-Bolaños E, Carrillo-Rodríguez J. Medicinal Plants Used by Indigenous Communities of Oaxaca, Mexico, to Treat Gastrointestinal Disorders. En: Perveen S, Al-Taweel A, editores. Pharmacognosy - Medicinal Plants: IntechOpen; 2019;1-36.

17. Choi JY, Ryoo E, Jo JH, Hann T, Kim SM. Risk factors of delayed diagnosis of acute appendicitis in children: for early detection of acute appendicitis. Korean J Pediatr. 2016;59(9):368-73.

18. Wong-Baker FACES Foundation (2018). Wong-Baker FACES ${ }^{\circledR}$ Pain Rating Scale. Retrieved. [15/11/2020] http://www. WongBakerFACES.org.

19. Glass CC, Rangel SJ. Overview and diagnosis of acute appendicitis in children. Semin Pediatr Surg. 2016;25(4):198-203.

20. Podany AB. Acute Appendicitis in Pediatric Patients: An Updated Narrative Review. J Clin Gastroenterol Treat. 2017;3(1):042:1-9.

21. Welton M, Rodríguez-Lainz A, Loza O, Brodine S, Fraga M. Use of lead- glazed ceramic ware and lead-based folk remedies in a rural community of Baja California, Mexico. Glob Health Promot. 2018;25(1):6-14.

22. Sandberg JC, Quandt SA, Graham A, Stub T, Mora DC, Arcury TA. Medical Pluralism in the Use of Sobadores among Mexican Immigrants to North Carolina. J Immigr Minor Health. 2018;20(5):1197205.

23. Marzuillo P, Germani C, Krauss BS, Barbi E. Appendicitis in children less than five years old: A challenge for the general practitioner. World J Clin Pediatr. 2015;4(2):19-21.

24. Serres SK, Cameron DB, Glass CC, Graham DA, Zurakowski D, Karki M, et al. Time to Appendectomy and Risk of Complicated Appendicitis and Adverse Outcomes in Children. JAMA Pediatr. 2017;171(8):740-6.

25. Marmo AS, editor. Appendicitis: Risk factors, management strategies and clinical implications. Emergency and intensive care medicine. New York: Nova Biomedical 2014; xvii: 296 pages.

26. Amin P, Cheng D. Management of Complicated Appendicitis in the Pediatric Population: When Surgery Doesn't Cut It. Semin Intervent Radiol. 2012;29(3):231-6.

27. Chen C-L, Chao H-C, Kong M-S, Chen S-Y. Risk Factors for Prolonged Hospitalization in Pediatric Appendicitis Patients with Medical Treatment. Pediatr Neonatol. 2017;58(3):223-8.

28. Khan J, Ali A, Sarwar B. Causes of Delayed presentation of acute appendicitis and how it affects morbidity and mortality. JSMC 2018;8:34-7.

29. Zvizdic Z, Pasic-Sefic I, Vranic S. Acute perforated appendicitis after blunt abdominal trauma: A report from a 7-year-old. Am J Emerg Med. 2020;38(2):408.e1-408.e2 